\title{
The Clinical Effectiveness of Using Kinesio Tape Following Wisdom Teeth Extraction
}

Sarah Henderson*, Jenny Alexanders and Thomas Shaw

Sport, Health and Sciences Department, The University of Hull, Washburn Building, Cottingham Road, Hull, United Kingdom

\begin{abstract}
Surgical removal of third molars $(3 \mathrm{M})$ is a routine, low risk procedure. Common post-surgical symptoms are typically swelling, pain and trismus. Kinesiology Taping (KT) is a relatively new treatment option in musculoskeletal pathologies and some of the hypotheses of KT is that it reduces pain, oedema and increases blood flow; however current evidence does not support its use. Patient A underwent a routine removal of 3 third molars via general anaesthetic. A post-surgical lymphatic drainage KT application was applied to the patient's lower mandible and masseter region. Results showed a significant reduction in pain, swelling and trismus was also reduced. The post op recovery time was decreased from the post op material of 10 days to 5 days; therefore suggesting that KT is potentially an effective treatment following surgical removal of wisdom teeth.
\end{abstract}

Keywords: Kinesio tape; Wisdom teeth extraction; Pain management; Post-surgical intervention.

\section{Introduction}

Removal of third molars $(3 \mathrm{M})$ is a routine procedure that is performed daily within dentistry [1,2] affecting over 75,000 patients in the UK per year [3] and up to 5 million in the United States [2]. Research has demonstrated that common symptoms associated with $3 \mathrm{M}$ are namely pain, swelling, discomfort and morbidity being the most common symptoms [1-3]. Currently, there are a number of approaches including advances in dentistry surgical tools, analgesics and hands on techniques which aim to reduce the post-operative morbidity, oedema and trismus, conversely with no significant difference. Consequently, new approaches need to be considered to effectively reduce these postoperative symptoms. Kinesio Taping (KT) has become a prevalent therapeutic tool in musculoskeletal (MSK), neurological and lymphatic conditions [4-8]. KT originated in the 1970's by Kenso Kase [9,10]. Subsequent scientific research states that KT can improve blood flow and lymphatic drainage by removing lymphatic fluid and haemorrhages [10] however not all current evidence supports its use and thus far there have been no high quality studies to outline its physiological effects. It is therefore plausible that KT has the potential to reduce morbidity, trismus and oedema; of which this case study supports.

\section{Case}

Patient A (Female, 26) presented with 3M's which was for an elective surgical extraction all of which were impacted, $23 \mathrm{M}$ were lingual and 1 was palatal. Patient A had suffered with wisdom teeth pain for 3 years with subsequent extraction aiming to decrease symptoms. Postoperative discussions between Patient $\mathrm{A}$ and the consultant revealed that it was highly likely that Patient A would suffer from severe oedema, pain and trismus. Patient A was placed under general anaesthetic to complete the extractions. Patient A had no previous medical conditions and was a healthy non-smoker.

Following two consultations, Patient A was admitted to receive extraction of three $3 \mathrm{M}$ due to all being impacted meaning they do not have enough room to emerge or develop normally. Pain scores were measured using a subjective 10 level visual analogue scale (VAS) where 0 indicates no pain, 5 moderate pain and 10, severe pain, these were taken pre and post operatively and then every day post operatively. Trismus was measured using a tape measure and was measured daily post operatively. Facial oedema was measured using a five-line measurement again measured daily [11]. The measurements which were taken for facial oedema are as stated in [11] "Line 1 was the most posterior point of the tragus to the most lateral point of the lip commissure, line 2 was the most posterior point of the tragus to the pogonium, line 3 was the most posterior point of the tragus to the lateral canthus of the eye, line 4 was the lateral canthus of the eye to the most inferior point angle of the mandible, and line 5 the most inferior point angle of the mandible to the midpoint of the nasal bone".

Kinesio Tex Gold" (KTG) was applied immediately after Patient A had come around from the general anaesthetic by a Kinesio Taping Practitioner (KTP) assisted by a Certified Kinesio Taping Practitioner (CKTP) (Figure 1). KTG was measured from Patient A's supraclavicular to the point of maximum swelling into 3 strips. KTG followed the lymphatic and circulatory protocols devised by Kase $[4,10]$ using $10 \%$ tension, directed to the supraclavicular nodes and gently rubbed to activate the adhesive and remained on Patient A for 5 days. A change of tape was not necessary. Patient A was also prescribed an antibiotic which needed to be taken daily which is standard protocol following invasive surgery [1] and codeine $30 \mathrm{mg}$ if required. The application effects aimed to reduce oedema, decrease trismus and pain by the physiological outcomes outlined by Kase $[4,10]$. Patient A had no side effects of the KTG.

The success of the treatment was assessed by measurements of trismus, pain and oedema; furthermore, photographs were also taken as a visual and comparative guide for the patient (Figure 1-3). Table 1 shows values for Trismus, Pain and Oedema. These were taken immediately post-surgery and continued for 7 days post-operative.

*Corresponding author: Sarah Henderson, Sport, Health and Sciences Department. The University of Hull, Washburn Building, Cottingham Road, Hull, HU67RX, United Kingdom, Tel: 07429421438; E-mail: S.henderson@hull.ac.uk

Received: December 21, 2015; Accepted: March 17, 2016; Published: March 24, 2016

Citation: Henderson S, Alexanders J, Shaw T (2016) The Clinical Effectiveness of Using Kinesio Tape Following Wisdom Teeth Extraction. Physiother Rehabil 1 : 103. doi: $10.4172 / 2573-0312.1000103$

Copyright: (c) 2016 Henderson S, et al. This is an open-access article distributed under the terms of the Creative Commons Attribution License, which permits unrestricted use, distribution, and reproduction in any medium, provided the original author and source are credited. 
Citation: Henderson S, Alexanders J, Shaw T (2016) The Clinical Effectiveness of Using Kinesio Tape Following Wisdom Teeth Extraction. Physiother Rehabil 1: 103. doi: 10.4172/2573-0312.1000103

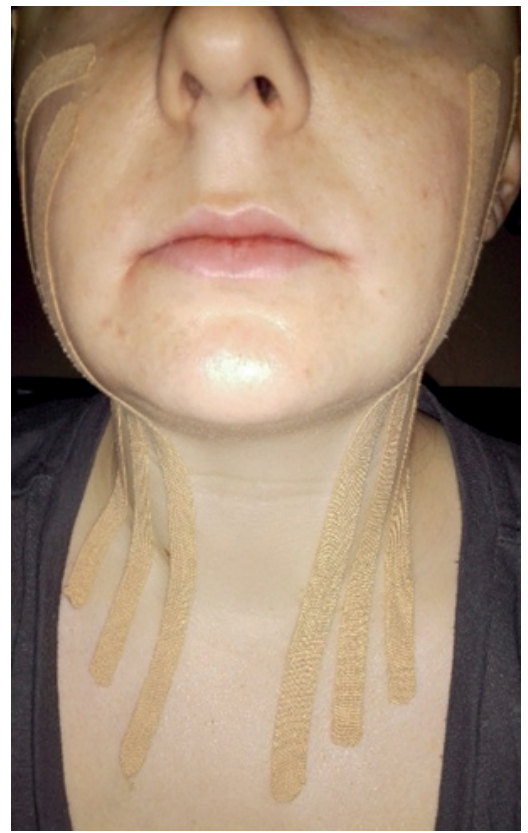

Figure 1: KTG application 6hours post-operative and facial oedema.

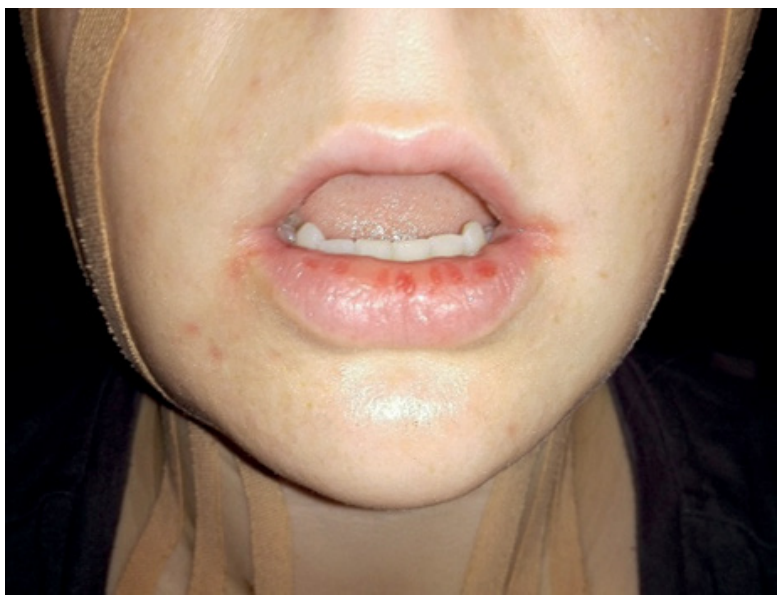

Figure 2: KTG application showing trismus 6hours post-operative.

\section{Discussion}

Thus far, only one study has investigated the use of KT with wisdom teeth extraction [11]. Numerous studies have researched the effects of KT with MSK conditions; however little investigations into the swelling aspects to Kase's hypothesis has yet to be explored. It has been concluded KT should be used for patients following wisdom teeth extraction following a clinical trial [11] and this case report supports this study; however further trials should be conducted to validate results further which could potentially inform post-operative protocols. With this type of surgery occurring regularly within dentistry, a review of post-surgical protocols should be addressed to enhance the patient's perceptions and experience and post-surgical care to accelerate recovery which could reduce days of sick taken by the patients, reducing costs. Due to the tapings simplistic application and lasting duration for lymphatic

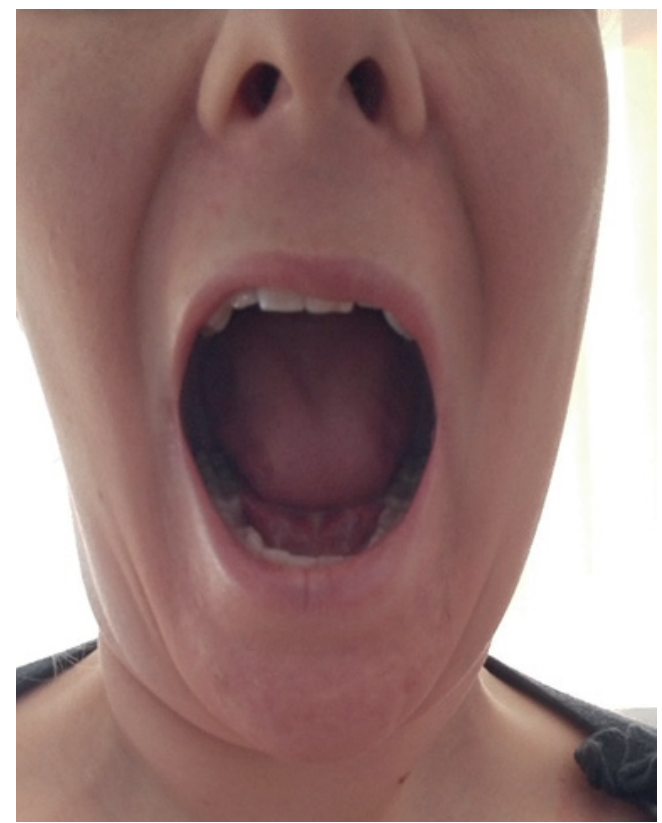

Figure 3: 5 days post-operative.

\begin{tabular}{|c|c|c|c|}
\hline & Trismus (cm) & Pain (VAS 0-10) & Oedema (cm) \\
\hline Pre-operative & $6 \mathrm{~cm}$ & 2 & N/A \\
\hline Post-operative day 1 & $1.5 \mathrm{~cm}$ & 5 & 1 \\
\hline $\mathbf{2}$ & $3.0 \mathrm{~cm}$ & 4 & 1.8 \\
\hline $\mathbf{3}$ & $4.0 \mathrm{~cm}$ & 3 & 1.5 \\
\hline $\mathbf{4}$ & $4.5 \mathrm{~cm}$ & 2 & 0.8 \\
\hline $\mathbf{5}$ & $6 \mathrm{~cm}$ & 0 & 0 \\
\hline $\mathbf{6}$ & $6 \mathrm{~cm}$ & 0 & 0 \\
\hline $\mathbf{7}$ & $6 \mathrm{~cm}$ & 0 & 0 \\
\hline
\end{tabular}

Table 1: Values for trismus, pain and oedema.

drainage, the application following extraction is economical, yet takes a medically pertinent approach.

\section{References}

1. BUPA (2015) Wisdom Teeth Removal.

2. Kalron A, Bar-Sela S (2013) A systematic review of the effectiveness of Kinesio Taping-fact or fashion? European journal of physical and rehabilitation medicine 49: 699-709.

3. Kase K, Hashimoto T, Tomoki O (1996) Development of Kinesio taping perfect manual. Kinesio Taping Association 6: 117-128.

4. Lim EC, Tay MG (2015) Kinesio taping in musculoskeletal pain and disability that lasts for more than 4 weeks: is it time to peel off the tape and throw it out with the sweat? A systematic review with meta-analysis focused on pain and also methods of tape application. British J of Spor Med 49: 1558-1566.

5. NHS (2015) Wisdom tooth removal.

6. NICE (2015) Guidance on the Extraction of Wisdom Teeth.

7. Morris D, Jones D, Ryan H, Ryan CG (2013) The clinical effects of Kinesio (R) Tex taping: A systematic review. Physiotherapy theory and practice 29: 259270.

8. Mostafavifar M, Wertz J, Borchers J (2012) A systematic review of the effectiveness of kinesio taping for musculoskeletal injury. The Physician and sports medicine 40 : 33-40.

9. Parreira PC, Costa LC, Hespanhol LC, Lopes AD, Costa LO (2014) Current evidence does not support the use of Kinesio Taping in clinical practice: a 
Citation: Henderson S, Alexanders J, Shaw T (2016) The Clinical Effectiveness of Using Kinesio Tape Following Wisdom Teeth Extraction. Physiother Rehabil 1: 103. doi: 10.4172/2573-0312.1000103

Page 3 of 3

systematic review. Journal of Physiotherapy 60: 31-39.

10. Kase K, Wallis J, Kase T (2003) 2nd edition. Clinical therapeutic applications of the Kinesio taping method, Japan.
11. Ristow O, Hohlweg-Majert B, Stürzenbaum SR, Kehl V, Koerdt S, et al. (2014) Therapeutic elastic tape reduces morbidity after wisdom teeth removal-a clinical trial. Clinical Oral Investigations 18: 1205-1212. 